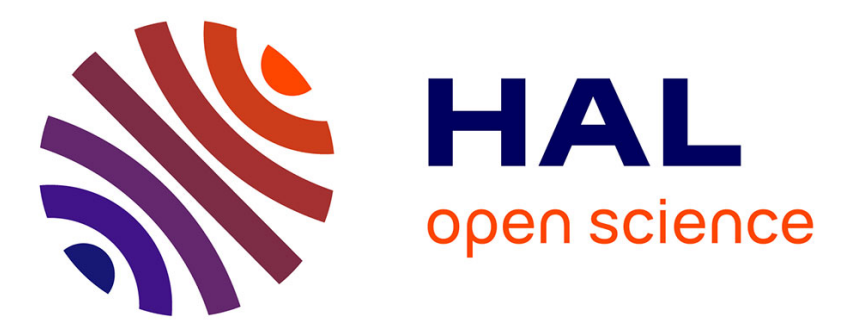

\title{
Creeping wave model of Diffraction of an Obliquely Incident Plane Wave by a Circular Cylinder at $60 \mathrm{GHz}$
}

Theodoros Mavridis, Luca Petrillo, Julien Sarrazin, David Lautru, Aziz Benlarbi-Delai, Philippe de Doncker

\section{- To cite this version:}

Theodoros Mavridis, Luca Petrillo, Julien Sarrazin, David Lautru, Aziz Benlarbi-Delai, et al.. Creeping wave model of Diffraction of an Obliquely Incident Plane Wave by a Circular Cylinder at $60 \mathrm{GHz}$. IEEE Transactions on Antennas and Propagation, 2014, 62 (3), pp.1372 - 1377. 10.1109/TAP.2013.2296321 . hal-00958276

\section{HAL Id: hal-00958276 https: / hal.sorbonne-universite.fr/hal-00958276}

Submitted on 12 Mar 2014

HAL is a multi-disciplinary open access archive for the deposit and dissemination of scientific research documents, whether they are published or not. The documents may come from teaching and research institutions in France or abroad, or from public or private research centers.
L'archive ouverte pluridisciplinaire HAL, est destinée au dépôt et à la diffusion de documents scientifiques de niveau recherche, publiés ou non, émanant des établissements d'enseignement et de recherche français ou étrangers, des laboratoires publics ou privés. 


\section{Creeping wave model of Diffraction of an Obliquely Incident Plane Wave by a Circular Cylinder at $60 \mathrm{GHz}$}

Theodoros Mavridis, Luca Petrillo, Julien Sarrazin, David Lautru, Aziz Benlarbi-Delaï, and Philippe De Doncker

\begin{abstract}
This paper presents a creeping wave model for the diffraction of an obliquely incident plane wave by a perfectly conducting or lossy circular cylinder at $60 \mathrm{GHz}$. The model developed for both TM and TE polarizations is valid for electrically large cylinders and for a receiver in the close vicinity of the surface. An experimental validation is conducted on a perfectly conducting cylinder.
\end{abstract}

\section{INTRODUCTION}

The high frequency scattering by a convex cylinder has been widely investigated in the past decades. Creeping wave theories have been developed [1], [2] in most cases for a normal incident plane wave.

Later, the Uniform Theory of Diffraction (UTD) [2], [3] have generalized those solutions to all kind of convex bodies and incident waves. The important property of UTD is the spatial continuity of the solution, regardless the position around the cylinder. But it necessitates heavy calculations of Fock functions [3]. In this context, this paper proposes a fast and accurate solution of the diffraction of a plane wave by a circular cylinder at $60 \mathrm{GHz}$ in the shadow region. This solution is based on known creeping wave models generalized to a 3D geometry and reduced to a simple path gain avoiding the computation of UTD.

In the Body Area Networks context [4], this solution is well suited for the calculation of the received power from an external transmitter to a receiver located on the body in the shadow region. In this paper, the results will be presented for both PEC cylinders and dielectric cylinders having the electric properties of the human skin.

The proposed model generalizes the model in [4] which is only suitable to normal incidence. Section II presents the analytical formulation for arbitrary incidence angle. In the shadow region, the scattered field is approximated as a creeping wave. Both TM and TE polarizations are considered. Section III validates our model by numerical comparisons with the exact solution and gives some values of the path gain factor of the creeping wave.

T. Mavridis, L. Petrillo and P. De Doncker are with OPERA Dpt. Wireless Communications Group, Université Libre de Bruxelles (ULB), B1050 Brussels, Belgium e-mail: (tmavridi@ulb.ac.be ; lpetrillo@ulb.ac.be ; pdedonck@ulb.ac.be)

J. Sarrazin, D. Lautru and A. Benlarbi-Delaï are with L2E Dept., Université Pierre et Marie Curie (UPMC), F-75005 Paris, France e-mail: (julien.sarrazin@upmc.fr; david.lautru@upmc.fr; aziz.benlarbi_delai@umpc.fr)
In section IV the model is compared to experimental results for both polarizations. The measurements have been conducted on a metallic cylinder for different elevation angles.

\section{Analytical Model}

\section{A. Problem Definition}

The geometry under study is drawn in Fig. 1.

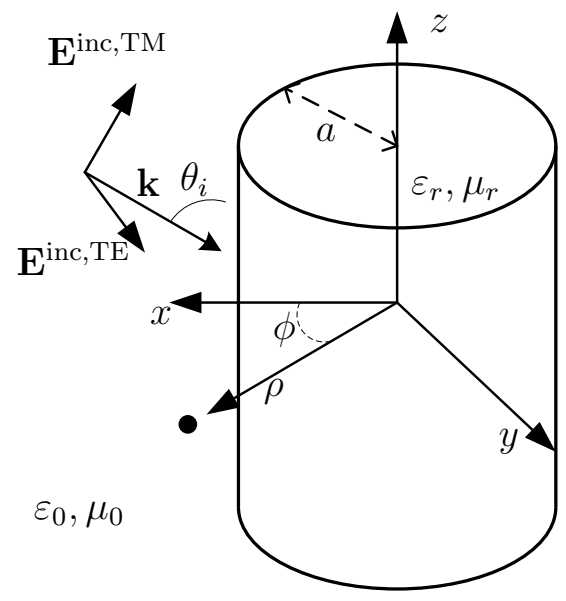

Fig. 1. Problem geometry.The wave vector $\mathbf{k}$ is assumed to lie in the plane $x z$. The incident electric field $\mathbf{E}$ lies in the plane $x z$ for TM polarization while it is polarized along $y$-axis for TE polarization.

The cylinder has a radius $a$, a principal axis $\hat{z}$, relative permeability $\mu_{r}$ and relative complex permittivity $\varepsilon_{r}$ depending on the conductivity $\sigma$ by $\varepsilon_{r}=\varepsilon_{r}^{\prime}+\frac{\sigma}{j \omega \varepsilon_{0}}$ with $\varepsilon_{r}^{\prime}$ defined as the real part of the relative permittivity and $\varepsilon_{0}, \mu_{0}$ are the free-space permittivity and permeability, respectively. A time dependence $e^{j \omega t}$ is assumed and suppressed, where $\omega=2 \pi f$ is the angular frequency, and $f$ is the frequency. Cylindrical coordinates $(\rho, \phi, z)$ are adopted where $-\pi \leq \phi \leq \pi, \rho>a$ and $-\infty \leq z \leq \infty$. In the following, the solution will be derived for positive values of $\phi$. By symmetry, the conclusions are exactly the same for negative values of $\phi$. Also, in this paper, ' defines the first derivative of a function.

The incident electric field $\mathbf{E}_{\text {inc }}$ for TM polarization is defined as:

$$
\mathbf{E}^{\mathrm{inc}, \mathrm{TM}}=E_{0}\left(\cos \theta_{i} \hat{x}+\sin \theta_{i} \hat{z}\right) e^{j k x \sin \theta_{i}} e^{j k z \cos \theta_{i}}
$$

where $E_{0}$ is the electric field amplitude, $k=\omega / c$ is the freespace wavenumber, $c$ is the speed of light and $\theta_{i}$ the elevation 
angle defined as $0 \leq \theta_{i} \leq \pi$.

In case of TE polarization, the incident electric field can be written as:

$$
\mathbf{E}^{\mathrm{inc}, \mathrm{TE}}=E_{0} e^{j k x \sin \theta_{i}} e^{j k z \cos \theta_{i}} \hat{y}
$$

The total electric field $\mathbf{E}^{\text {tot }}$ around the cylinder is:

$$
\mathbf{E}^{\text {tot }}=\mathbf{E}^{\text {inc }}+\mathbf{E}^{\mathrm{s}}
$$

where $\mathbf{E}^{\mathrm{s}}$ is the scattered field.

This paper focuses on the diffracted field in the shadow region which is defined geometrically by $\phi>\phi_{\mathrm{b}}=\pi / 2+$ $\operatorname{acos}\left(\frac{a}{\rho}\right)$ in [5]. In the following, the field is calculated on the range $\phi \in[\pi / 2, \pi]$.

\section{B. TM polarization}

The exact solution of the scattering of a plane wave by a dielectric cylinder is given in a lossless case in [6] and can be generalized to a lossy one:

$$
\begin{aligned}
& E_{\rho}^{\mathrm{s}}=-j E_{0} \cos \theta_{i} e^{j k z \cos \theta_{i}} \sum_{n=-\infty}^{+\infty} j^{n} a_{n} H_{n}^{(2)^{\prime}}\left(k \rho \sin \theta_{i}\right) e^{-j n \phi} \\
& E_{\phi}^{\mathrm{s}}=E_{0} \frac{\cot \theta_{i}}{j k \rho} e^{j k z \cos \theta_{i}} \sum_{n=-\infty}^{+\infty} n j^{n-1} a_{n} H_{n}^{(2)}\left(k \rho \sin \theta_{i}\right) e^{-j n \phi} \\
& E_{z}^{\mathrm{s}}=E_{0} \sin \theta_{i} e^{j k z \cos \theta_{i}} \sum_{n=-\infty}^{+\infty} j^{n} a_{n} H_{n}^{(2)}\left(k \rho \sin \theta_{i}\right) e^{-j n \phi}
\end{aligned}
$$

It can be shown that $a_{n}$ can be written in the high frequency range [2] as:

$$
a_{n}=-\frac{J_{n}^{\prime}\left(k a \sin \theta_{i}\right)+Q J_{n}\left(k a \sin \theta_{i}\right)}{H_{n}^{(2)^{\prime}}\left(k a \sin \theta_{i}\right)+Q H_{n}^{(2)}\left(k a \sin \theta_{i}\right)}
$$

where $J_{n}$ and $H_{n}^{(2)}$ are, respectively, the first kind Bessel functions and the second order Hankel functions [7] and $Q=j \eta, \eta=\sqrt{\varepsilon_{r} / \mu_{r}}$ is the normalized admittance with respect to the free space admittance $\sqrt{\varepsilon_{0} / \mu_{0}}$ [8] and $k=k_{0}$.

In (4), at high frequency, the series slowly converge and the number of terms required for convergence is too large to allow a fast computation. To speed up convergence in the shadow region, we propose a simplified model where the electric field is approximated by a creeping wave.

By using Watson's transformation [9] on (4), the total field in the region $\phi>\pi / 2$ becomes:

$$
\begin{aligned}
& E_{\rho}^{\mathrm{tot}}=-\frac{2 \pi}{m} j E_{0} \cos \theta_{i} e^{j k z \cos \theta_{i}} \sum_{s} j^{v_{s}} \tilde{a}_{v_{s}} W_{2}{ }^{\prime}\left(\tau_{s}-h\right) \Phi_{v_{s}}(\phi) \\
& E_{\phi}^{\mathrm{tot}}=2 \pi E_{0} \frac{\cot \theta_{i}}{j k \rho} e^{j k z \cos \theta_{i}} \sum_{s} v_{s} j^{v_{s}-1} \tilde{a}_{v_{s}} W_{2}\left(\tau_{s}-h\right) \Phi_{v_{s}}(\phi) \\
& E_{z}^{\mathrm{tot}}=2 \pi E_{0} \sin \theta_{i} e^{j k z \cos \theta_{i}} \sum_{s} j^{v_{s}} \tilde{a}_{v_{s}} W_{2}\left(\tau_{s}-h\right) \Phi_{v_{s}}(\phi)
\end{aligned}
$$

where $m=\left(k a \sin \theta_{i}\right)^{1 / 3}, v_{s}=k a \sin \theta_{i}+m \tau_{s}$ and:

$$
\begin{aligned}
\Phi_{v}(\phi) & =\frac{\cos (v(\phi-\pi))}{\sin (v \pi)} \\
\tilde{a}_{v_{s}} & =\frac{A^{\prime}\left(\tau_{s}\right)-q A\left(\tau_{s}\right)}{\tau_{s} W_{2}\left(\tau_{s}\right)-q W_{2}^{\prime}\left(\tau_{s}\right)} \\
h & =\frac{k}{m} \sin \theta_{i}(\rho-a) .
\end{aligned}
$$

Equations (6) and (7) have been obtained by using Olver's transformation [2], [10] which allows to write Bessel's function in terms of Airy's:

$$
\begin{aligned}
J_{v}\left(k a \sin \theta_{i}\right) & \approx \frac{1}{m} A(\tau) \\
H_{v}^{(2)}\left(k a \sin \theta_{i}\right) & \approx \frac{-j}{m \sqrt{\pi}} W_{2}(\tau) \\
J_{v}\left(k \rho \sin \theta_{i}\right) & \approx \frac{1}{m} A(\tau-h) \\
H_{v}^{(2)}\left(k \rho \sin \theta_{i}\right) & \approx \frac{-j}{m \sqrt{\pi}} W_{2}(\tau-h)
\end{aligned}
$$

This transformation implies two conditions. First, on the argument of the Bessel function: $k a \sin \theta_{i} \gg 1$. This condition implies a conditional relationship between $a$ and $\theta_{i}$. For instance, in the BAN context, the radius of the cylinder $a$ is fixed and it infers that $\sin \theta_{i} \gg 1 / k a$. Secondly, a close vicinity assumption has to be made for $\rho$. This is easily obtained by assuming $a<\rho<2 a$. Considering a maximum error of $3 \mathrm{~dB}$ on $E$, it can be numerically obtained that $a<\rho<1.2 a$.

In those equations, $q=m Q$ and the $\tau_{s}$ are defined by:

$$
W_{2}^{\prime}\left(\tau_{s}\right)-q W_{2}\left(\tau_{s}\right)=0
$$

$A(z)$ is the first kind Airy function and $W_{2}(z)=$ $2 e^{j \pi / 6} A\left(e^{j 4 \pi / 3} z\right)$. At $60 \mathrm{GHz}$ and knowing the condition on the imaginary parts $\left|\operatorname{Im}\left(v_{1}\right)\right| \ll\left|\operatorname{Im}\left(v_{s}\right)\right|$ with $s>2$, the sum (6) can be approximated by:

$$
\begin{aligned}
& E_{\rho}^{\mathrm{tot}}=-\frac{2 \pi}{m} j E_{0} \cos \theta_{i} e^{j k z \cos \theta_{i}} j^{v_{1}+1} \tilde{a}_{v_{1}} W_{2}{ }^{\prime}\left(\tau_{1}-h\right) e^{-j v_{1} \phi} \\
& E_{\phi}^{\mathrm{tot}}=-2 \pi j E_{0} \frac{\cot \theta_{i}}{k \rho} e^{j k z \cos \theta_{i}} v_{1} j^{v_{1}} \tilde{a}_{v_{1}} W_{2}\left(\tau_{1}-h\right) e^{-j v_{1} \phi} \\
& E_{z}^{\mathrm{tot}}=2 \pi E_{0} \sin \theta_{i} e^{j k z \cos \theta_{i}} j^{v_{1}+1} \tilde{a}_{v_{1}} W_{2}\left(\tau_{1}-h\right) e^{-j v_{1} \phi}
\end{aligned}
$$

by using $\Phi_{v_{s}}(\phi) \simeq j e^{-j v_{s} \phi}$. Equation (10) gives the creeping wave solution in the shadow zone.

The path gain of the creeping wave can be inferred by introducing the power $P_{\text {tot }}=20 \log _{10}\left|\mathbf{E}^{\text {tot }}\right|$ expressed in dB:

$$
P_{\gamma}=P_{\gamma}^{\pi / 2}+n^{\mathrm{TM}}(\pi / 2-\phi)
$$

where $\gamma=\rho, \phi, z$ and $P_{\gamma}^{\pi / 2}$ is the received power at the boundary of the shadow zone $\phi=\pi / 2$ for the $\gamma$ component. It is remarkable that the path gain factor $n^{\mathrm{TM}}$ is the same for each component. The path gain $P_{\text {tot }}$ for the total field can also be easily inferred:

$$
P_{\mathrm{tot}}=P_{\mathrm{tot}}^{\pi / 2}+n^{\mathrm{TM}}(\pi / 2-\phi)
$$

The path gain factor can be derived from (10):

$$
n^{\mathrm{TM}}=20 \log _{10}(e)\left|\operatorname{Im}\left(\tau_{1}\right)\right| m
$$




\section{TE polarization}

For TE polarization, the incident electric field is:

$$
\begin{aligned}
& E_{\rho}^{\mathrm{inc}}=-E_{0} \sin \phi e^{j k z \cos \theta_{i}} e^{j k x \sin \theta_{i}} \\
& E_{\phi}^{\mathrm{inc}}=-E_{0} \cos \phi e^{j k z \cos \theta_{i}} e^{j k x \sin \theta_{i}} \\
& E_{z}^{\mathrm{inc}}=0
\end{aligned}
$$

The exact solution can also be easily obtained by solving the boundary conditions problem in cylindrical coordinates:

$$
\begin{aligned}
& E_{\rho}^{\mathrm{s}}=-j \frac{E_{0}}{k \rho} \frac{e^{-j k z \cos \theta_{i}}}{\sin \theta_{i}} \sum_{n=-\infty}^{+\infty} n j^{n-1} b_{n} H_{n}^{(2)}\left(k \rho \sin \theta_{i}\right) e^{-j n \phi} \\
& E_{\phi}^{\mathrm{s}}=j E_{0} e^{-j k z \cos \theta_{i}} \sum_{n=-\infty}^{+\infty} j^{n} b_{n} H_{n}^{(2)^{\prime}}\left(k \rho \sin \theta_{i}\right) e^{-j n \phi} \\
& E_{z}^{\mathrm{s}}=0
\end{aligned}
$$

where, by using the high frequency assumption:

$$
b_{n}=-\frac{Q J_{n}^{\prime}\left(k a \sin \theta_{i}\right)+J_{n}\left(k a \sin \theta_{i}\right)}{Q H_{n}^{(2)^{\prime}}\left(k a \sin \theta_{i}\right)+H_{n}^{(2)}\left(k a \sin \theta_{i}\right)}
$$

Equations (3) is used to get the total field for each component. The same methodology as used in section II-B is then carried out to speed up convergence.

By applying Watson's transformation, the creeping wave expression of the total field in the shadow region is:

$$
\begin{aligned}
& E_{\rho}^{\text {tot }}=-j \frac{E_{0}}{k \rho} \frac{e^{-j k z \cos \theta_{i}}}{\sin \theta_{i}} 2 \pi v_{1}^{\prime} j^{v_{1}^{\prime}} \tilde{b}_{v_{1}^{\prime}} W_{2}\left(\tau_{1}^{\prime}-h\right) e^{-j v_{1}^{\prime} \phi} \\
& E_{\phi}^{\text {tot }}=j E_{0} e^{-j k z \cos \theta_{i}} \frac{2 \pi}{m} j^{v_{1}^{\prime}+1} \tilde{b}_{v_{1}^{\prime}} W_{2}^{\prime}\left(\tau_{1}^{\prime}-h\right) e^{-j v_{1}^{\prime} \phi} \\
& E_{z}^{\text {tot }}=0
\end{aligned}
$$

where $v_{1}^{\prime}=k a \sin \theta_{i}+m \tau_{1}^{\prime}$ and $\tau_{1}^{\prime}$ is the first zero of:

$$
q W_{2}^{\prime}\left(\tau_{1}^{\prime}\right)-W_{2}\left(\tau_{1}^{\prime}\right)=0
$$

and

$$
\tilde{b}_{v_{1}^{\prime}}=\frac{q A^{\prime}\left(\tau_{1}^{\prime}\right)-A\left(\tau_{1}^{\prime}\right)}{q \tau_{1}^{\prime} W_{2}\left(\tau_{1}^{\prime}\right)-W_{2}^{\prime}\left(\tau_{1}^{\prime}\right)} .
$$

These equations give the path gain:

$$
P_{\text {tot }}=P_{\text {tot }}^{\pi / 2}+n^{\mathrm{TE}}(\pi / 2-\phi)
$$

where $P_{\text {tot }}=20 \log _{10}\left|\mathbf{E}^{\text {tot }}\right|$ in $\mathrm{dB}$, and

$$
n^{\mathrm{TE}}=20 \log _{10}(e)\left|\operatorname{Im}\left(\tau_{1}^{\prime}\right)\right| m .
$$

The path gain of each component of the electric field can be rewritten as (11) by using $n^{\mathrm{TE}}$ for the path gain factor.

\section{NUMERICAL SIMULATIONS}

Two configurations have been studied: the perfectly conducting cylinder (PEC) and the dielectric cylinder having the electric properties of the human skin [11] $\left(\varepsilon_{r}^{\prime}=7.9753, \sigma=\right.$ $36.397 \mathrm{~S} / \mathrm{m}$ ). The first zeroes $\tau_{1}, \tau_{1}^{\prime}$ are computed from (9) and (18).

\section{A. TM polarization}

In the TM case, the dependence of $\tau_{1}$ with the elevation angle $\theta_{i}$ is negligible. The solution of (9) gives $\tau_{1}=1.17-$ $j 2.025$ for the PEC cylinder, and $\tau_{1}=1.14-j 1.97$ for the dielectric case.

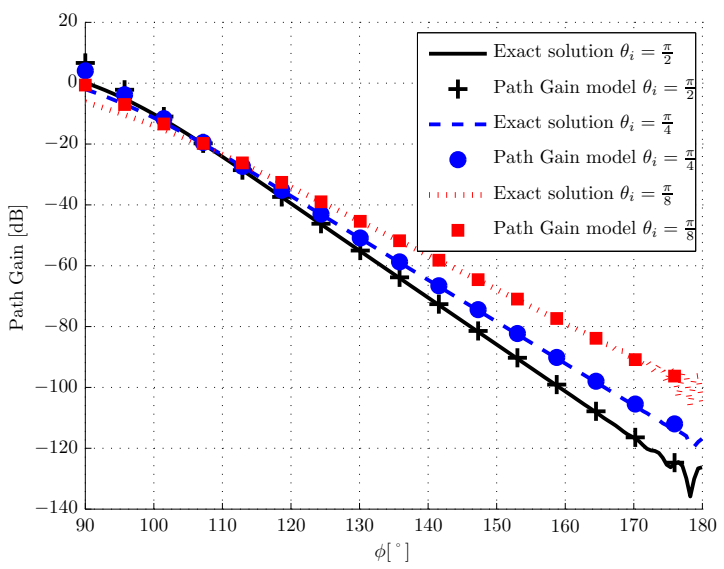

Fig. 2. Total Field in the shadow zone for TM polarization with $f=60 \mathrm{GHz}$, $a=20 \mathrm{~cm}$ and $\rho=20.5 \mathrm{~cm}$ for a PEC cylinder.

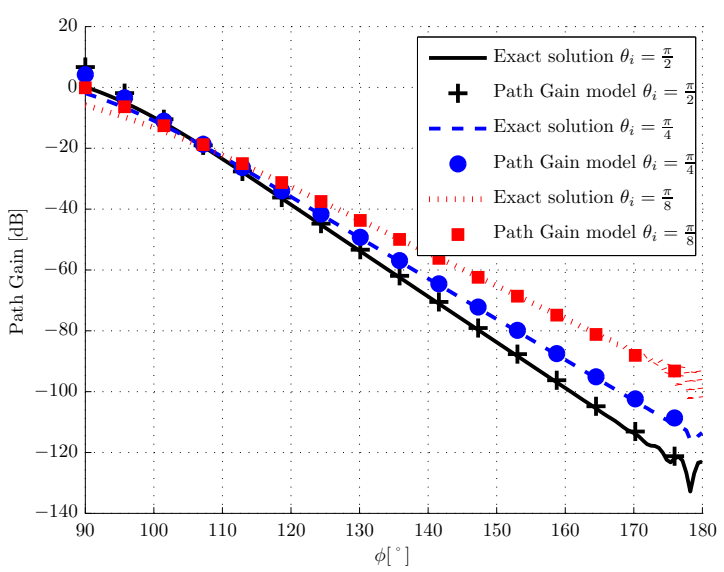

Fig. 3. Total Field in the shadow zone for TM polarization with $f=60 \mathrm{GHz}$, $a=20 \mathrm{~cm}$ and $\rho=20.5 \mathrm{~cm}$ for a cylinder with the properties of the human skin.

As can be seen in Figures 2 and 3, the creeping wave model fits the exact solution in the PEC and dielectric cases. The magnitudes have been normalized in order to have unitary incident electric field magnitude.

\section{B. TE polarization}

Figures 4 and 5 present the path gain for TE polarization for different values of $\theta_{i}$. It can be seen that the creeping wave model fits the exact solution in both cases. In the PEC case, $\tau_{1}^{\prime}=0.51-j 0.88$ does not depend on $\theta_{i}$. In the dielectric case, the variation of $\tau_{1}^{\prime}$ with $\theta_{i}$ has to be taken into account.

Table I summarizes the path gain factors in $[\mathrm{dB} / \mathrm{cm}]$ for different values of the radius $a$ and elevation angle $\theta_{i}$. It 


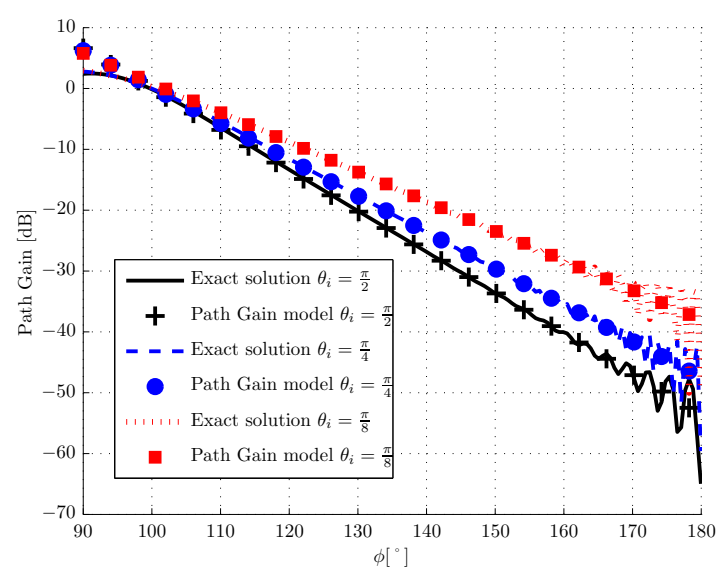

Fig. 4. Total Field in the shadow zone for TE polarization with $f=60 \mathrm{GHz}$, $a=20 \mathrm{~cm}$ and $\rho=20.5 \mathrm{~cm}$ for a PEC cylinder.

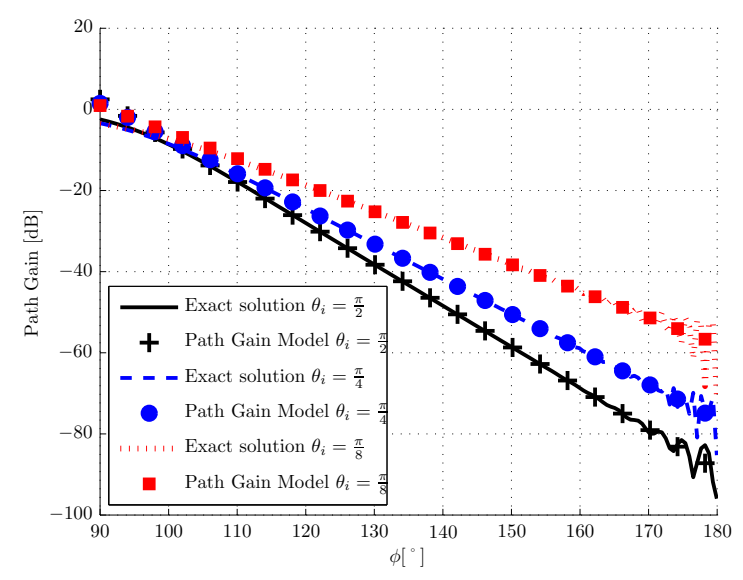

Fig. 5. Total Field in the shadow zone for TE polarization with $f=60 \mathrm{GHz}$, $a=20 \mathrm{~cm}$ and $\rho=20.5 \mathrm{~cm}$ for a cylinder with the properties of the human skin.

is worth noting that the path gain factor decreases with decreasing elevation angle $\theta_{i}$.

This analysis gives some useful insights regarding BAN applications. To improve off-body communications in terms of path gain using creeping wave modes, remote base stations should be located on the ceiling in order to minimize the elevation angle. Figures 2 - 5 confirm that the first creeping wave mode is predominant at $60 \mathrm{GHz}$. The influence of the creeping wave mode coming from the other side of the cylinder $(\phi<0)$ can be observed in the exact solutions around $\phi=180^{\circ}$ where an interference pattern appears. Given the low power level reached for these values of $\phi$, it can be assumed that this creeping wave mode is negligible.

\section{EXPERIMENTAL RESUlts}

Experiments have been performed on a PEC cylinder, allowing to obtain very accurate measurement of the path gain around the cylinder to discriminate path gain factors for different elevation angles $\theta_{i}$.
TABLE I

GAINS $\left(n^{\mathrm{TM}}, n^{\mathrm{TE}}\right)$ IN $[d \mathrm{~B} / \mathrm{CM}]$ FOR A PEC CYLINDER AND A DIELECTRIC CYLINDER FILLED WITH THE ELECTRIC PROPERTIES OF THE HUMAN SKIN.

\begin{tabular}{c|c|cccc}
\hline \hline \multicolumn{2}{c|}{} & \multicolumn{4}{c}{$\theta_{i}[\mathrm{rad}]$} \\
\hline \multicolumn{2}{c}{ PEC } & $\pi / 2$ & $\pi / 4$ & $\pi / 6$ & $\pi / 8$ \\
\hline \multirow{4}{*}{$a[\mathrm{~cm}]$} & 15 & $(5.34,2.32)$ & $(4.75,2.07)$ & $(4.23,1.84)$ & $(3.87,1.69)$ \\
& 20 & $(4.40,1.92)$ & $(3.92,1.71)$ & $(3.50,1.52)$ & $(3.20,1.39)$ \\
& 25 & $(3.80,1.65)$ & $(3.38,1.47)$ & $(3.01,1.31)$ & $(2.76,1.20)$ \\
& 30 & $(3.36,1.46)$ & $(2.99,1.30)$ & $(2.67,1.16)$ & $(2.44,1.06)$ \\
\hline \multicolumn{2}{c|}{ Dielectric } & $\pi / 2$ & $\pi / 4$ & $\pi / 6$ & $\pi / 8$ \\
\hline & 15 & $(5.20,3.40)$ & $(4.61,2.89)$ & $(4.10,2.47)$ & $(3.73,2.19)$ \\
$a[\mathrm{~cm}]$ & 20 & $(4.30,2.91)$ & $(3.82,2.50)$ & $(3.39,2.11)$ & $(3.09,1.87)$ \\
& 25 & $(3.71,2.58)$ & $(3.30,2.20)$ & $(2.93,1.87)$ & $(2.67,1.66)$ \\
& 30 & $(3.29,2.34)$ & $(2.92,2.00)$ & $(2.60,1.70)$ & $(2.37,1.50)$ \\
\hline \hline
\end{tabular}

The aim of this measurement campaign is to verify the azimuthal variation of the absolute level of the path gain for different elevation angles. Due to the radiation pattern of the antennas, the path gains for different elevation angles cannot be compared. Hence, the measurement and simulations have been arbitrarily normalized at $0,-15$ and $-30 \mathrm{~dB}$, respectively, for $\theta_{i}=90^{\circ}, 55^{\circ}$ and $25^{\circ}$ for sake of readability.

\section{A. Measurement Set-up}

The measurements were conducted with an Agilent E8361C $V N A$ and U-band horn antennas (20 dB gain) in an anechoic chamber. To increase the dynamic range, two amplifiers have been used (the first at the transmitter side and the second at the receiver side). The measurement parameters are summarized in Table II. The distance $d$ between the transmit antenna and the cylinder was fixed for each measurement to ensure a full exposition of the cylinder. The Rx horn antenna was placed tangentially to the cylinder to maximize the amount of power received from the creeping wave. The cylinder had a height of $1.2 \mathrm{~m}$ and the Rx horn antenna was placed at middle height.

TABLE II

MEASUREMENTS MAIN PARAMETERS

\begin{tabular}{cc}
\hline \hline Symbol & Value \\
\hline$\theta_{i}$ & $25^{\circ}, 55^{\circ}, 90^{\circ}$ \\
$a$ & $0.2 \mathrm{~m}$ \\
$d$ & $2 \mathrm{~m}$ \\
$\phi$ & $90^{\circ}-145^{\circ}$ \\
Angular Step $\Delta \phi$ & $5^{\circ}$ \\
$f_{\text {start }}$ & $50 \mathrm{GHz}$ \\
$f_{\text {stop }}$ & $60 \mathrm{GHz}$ \\
$f_{\text {step }}$ & $66.67 \mathrm{MHz}$ \\
VNA IFbandwidth & $1 \mathrm{~Hz}$ \\
VNA Averaging & 1024 \\
\hline \hline
\end{tabular}

The IFbandwidth and the VNA averaging were chosen to have the highest dynamic range possible. The measurement set-up is shown in Fig. 6.

The coaxial cables have about $6 \mathrm{~dB} / \mathrm{m}$ losses. To avoid the need of long distance cables and maximize the received power, the VNA was put inside the anechoic chamber and covered by absorbing material. 


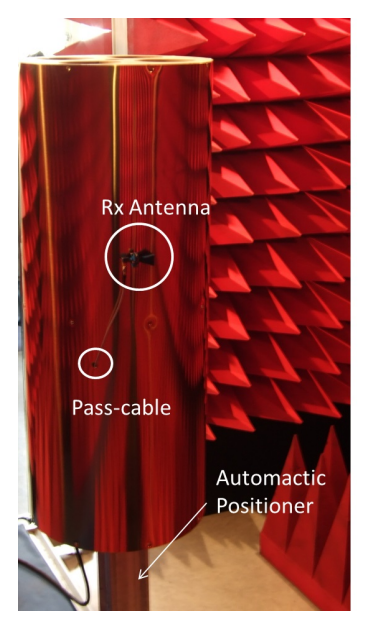

Fig. 6. Experimental set-up

\section{B. Measurement Results}

The measurements have been performed for both polarizations for three values of elevation angle. Time gating has been performed to increase the dynamic range. It was centered on the predicted time of arrival of the creeping wave.

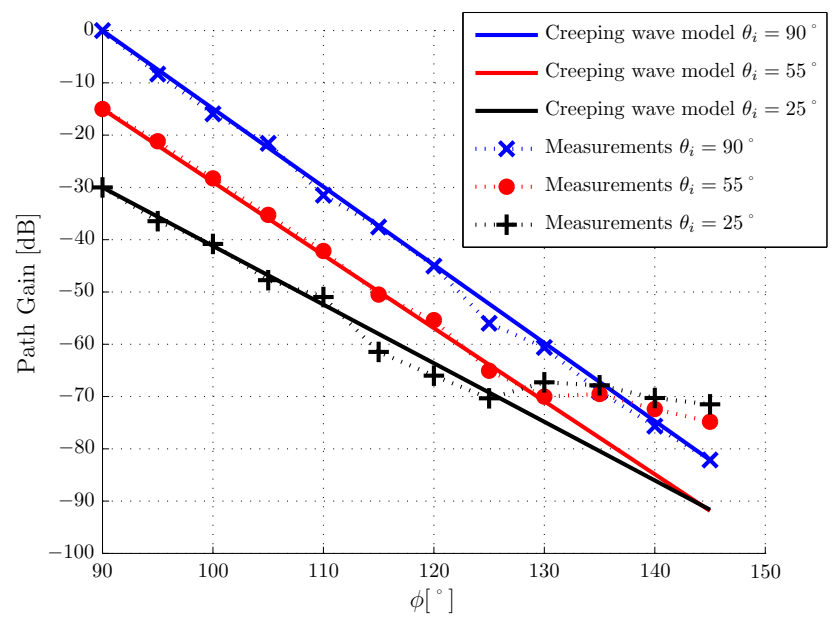

Fig. 7. Normalized Measurement Results for TM polarization and comparison with the creeping wave path gain.

The results in Fig. 7 and 8 show that the analytical creeping wave models (12) and (20) fit the measurements. As can be seen, the TE mode has a higher dynamic range and, consequently, measurements are obtained up to higher values of $\phi$.

\section{CONClusion}

The paper investigates the scattering of an obliquely incident plane wave by a circular cylinder at $60 \mathrm{GHz}$. The cylinder radius has been assumed electrically large. A propagation model has been developed for both TM and TE polarizations. The model assumes that the propagation can be described by a creeping wave in the shadow region. By using Watson's transformation, TM and TE creeping wave models

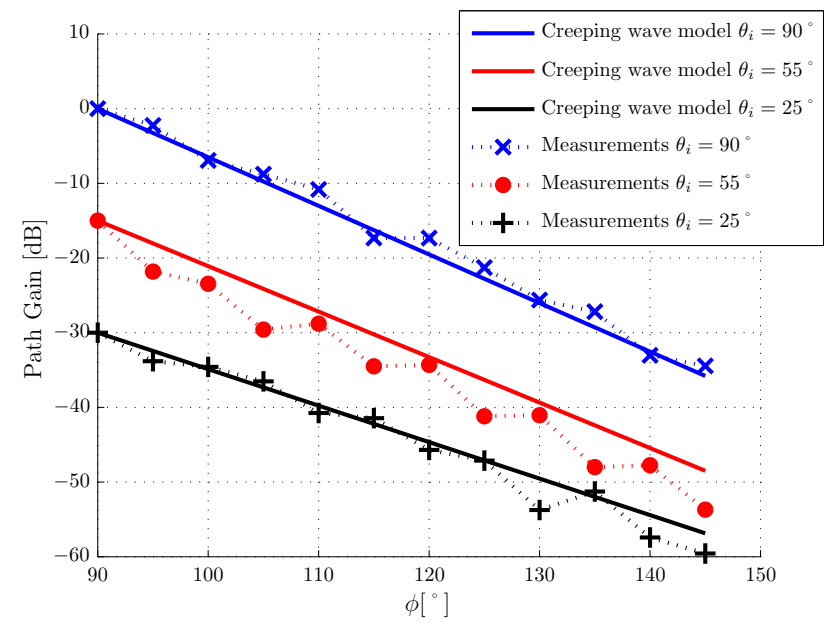

Fig. 8. Normalized Measurement Results for TE polarization and comparison with the creeping wave path gain

have been deduced.

Numerical simulations have been conducted to validate the creeping wave model. The simulations give the path gain factors in the shadow zone for a PEC cylinder and for a lossy cylinder filled with a dielectric having the electric properties of the human skin to emulate a Body Area Network scenario. The simulations have shown that the path gain factor decreases with decreasing elevation angle.

The experimental validation has been done on a PEC cylinder for both polarizations. The creeping wave path gain model developed in this paper fits the measurements.

\section{ACKNOWLEDGEMENT}

The authors would like to thank Prof. Bernard Huart and Antoine Khy from Telecom ParisTech for their helpful advises.

\section{REFERENCES}

[1] R. Paknys, "Uniform asymptotic formulas for the creeping wave field on or off a cylinder," IEEE Trans. Antennas Propag., vol. 41, no. 8, pp. 1099-1104, 1993.

[2] H. H. Syed and J. L. Volakis, "High frequency scattering by a smooth coated cylinder simulated with generalized impedance boundary conditions," Radio Sci., vol. 26, pp. 1305-1314, 1991.

[3] P. H. Pathak, W. D. Burnside, and R. J. Marhefka, "A uniform GTD analysis of the diffraction of electromagnetic waves by a smooth convex surface," IEEE Trans. Antennas Propag., vol. AP-28, no. 5, pp. 631-642, 1980.

[4] T. Mavridis, L. Petrillo, J. Sarrazin, D. Lautru, Benlarbi-Delaï, and P. De Doncker, "Theoretical and experimental investigation of a $60 \mathrm{GHz}$ off-body propagation model," IEEE Trans. Antennas Propag., Accepted.

[5] Y. A. Kravtsov and N. Y. Zhu, Theory of Diffraction : Heuristic Approaches. 2010: Alpha Science, 2010.

[6] W. C. Chew, Waves and Fields in Inhomogeneous Media. New York: IEEE Press, 1995.

[7] M. Abramowitz and I. A. Stegun, Handbook of Mathematical Functions with Formulas, Graphs, and Mathematical Tables. Dover, 1992.

[8] L. D. Landau and E. M. Lifshitz, Electrodynamics of Continous Media. Pergamon, 1984. 
[9] C. A. Valagiannopoulos, "An overview of the watson transformation presented through a simple example," Progress In Electromagnetics Research, PIER 75, pp. 137-152, 2007.

[10] F. W. Olver, "Asymptotics and special functions," New York: Academic, 1974.

[11] P. A. Hasgall, E. Neufeld, M.-C. Gosselin, A. Klingenböck, and N. Kuster, "IT'IS database for thermal and electromagnetic parameters of biological tissues, version 2.2," July 11th, 2012. [Online]. Available: http://www.itis.ethz.ch/database

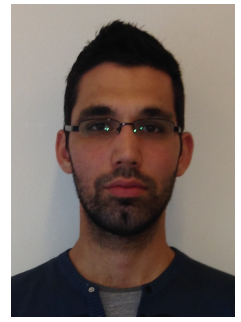

Theodoros Mavridis is born in Brussels, Belgium, in 1988. He received his Master degree in physical engineering from the Universit Libre de Bruxelles, Brussels, Belgium, in 2011. In 2011, he joined the OPERA Dpt. - Wireless Communication Group where he began his thesis on the propagation channel for Body Area Networks at $60 \mathrm{GHz}$ and the spatial focusing techniques.

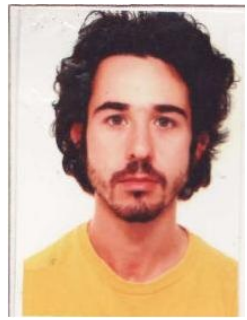

Luca Petrillo graduated in electronic engineering from the University of Genoa, Italy, in 2008. He received the Master's degree in Communication Systems and the Doctoral degree from the University Pierre et Marie Curie, Paris, France, in 2008 and 2011, respectively. From October 2008 to September 2011 he was a researcher at Onera, France. From October 2011 to August 2012 he was an Associate Professor at the University Pierre et Marie Curie, where he taught electronics. Since September 2012 he accomplishes post-doctoral training at the Ecole Polytechnique of Bruxelles, Belgium. His scientific interests are in the field of electromagnetic propagation, including surface wave propagation, periodic structures (metamaterials, EBG), human body propagation in different scenarios, and in electromagnetic systems, including HF radars, Body Area Networks, $60 \mathrm{GHz}$ wireless systems, Green Radio.

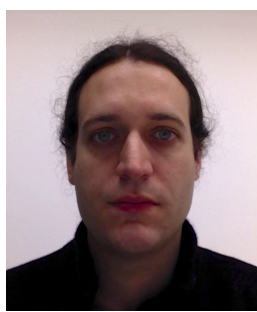

Julien Sarrazin received his Master and $\mathrm{PhD}$ degrees from the University of Nantes in France, in 2005 and 2008 respectively. In 2009 and 2010, he worked at the BK Birla Institute of Technology of Pilani in India. In 2011 and 2012, he was a research engineer at Telecom ParisTech in Paris. Since September 2012, he is Associate Professor at the University of Pierre and Marie Curie (UPMC) in Paris, where he is currently working in the Electronics and Electromagnetism Lab (L2E) in the field of Body Area Networks (BAN) and $60 \mathrm{GHz}$ communications. His research interests also include antenna design, MIMO systems, wireless communication and localization, propagation channel, metamaterials.

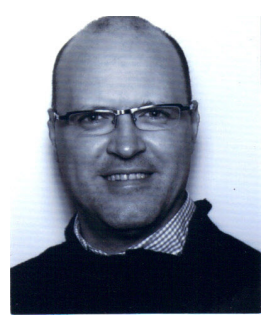

Aziz Benlarbi-Delaï received the $\mathrm{PhD}$ in Electrical Engineering and The Habilitation Diriger des Recherches (HDR) s Sciences Physiques from the University of Lille I in 1992 and 2002. From 1992 to 2006 he was Assistant Professor at this University and was involved, as researcher at the Institute of Electronic Microelectronic and Nanotechnology (IEMN), in the field of microwave and microfluidic devices and systems for connected objects, and also in the field of ultra fast sampling using micro and nanostructures. He is currently a full time Professor in Electrical Engineering at the University Pierre et Marie Curie (UPMC Paris 06) and head of the laboratory of Electronics and Electromagnetism (L2E). He is involved in research aiming millimetre wave communication and localization for green radio and intelligent ambient issues.

$\mathrm{He}$ is the author of 94 publications and communications and the holder of two patents. He participated to several Technical Program Committees of international conferences and is visiting professor or member of the external evaluation team of several foreign universities.

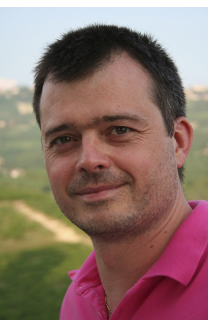

Philippe De Doncker received the M.Sc. in physics engineering and Ph.D. degrees from the Universit libre de Bruxelles (ULB), Belgium, in 1996 and 2001 , respectively. He is currently Professor with the Universit libre de Bruxelles. His research interests focus on wireless communications and electromagnetics.

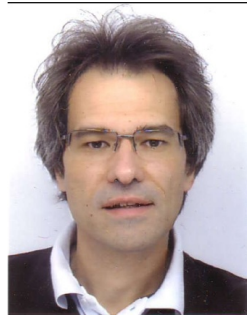

David Lautru received the $\mathrm{PhD}$ degree in Electrical Engineering and his Qualification to Lead Research HDR (Habilitation Diriger des Recherches) from the University Pierre and Marie Curie (UPMC), Paris, France, in 2000 and 2012 respectively.. He is currently an associate professor at the Laboratory of Electronics and Electromagnetism (L2E) of the UPMC. His fields of interest are electromagnetic modeling, computational electromagnetics and bioelectromagnetics. 